\title{
Factores asociados a escucha activa durante prácticas clínicas en estudiantes de enfermería de Selva peruana
}

Factors associated with active listening during clinical practices in nursing students in the Peruvian jungle

Jessica Ticliahuanca-Paredes ${ }^{1}$

\begin{abstract}
Resumen
Objetivo: determinar qué factores se asocian a la escucha activa en la práctica clínica de los estudiantes de enfermería de la Selva peruana. Materiales y métodos: el estudio fue descriptivo-correlacional de diseño transversal. La población estuvo conformada por 85 universitarios de enfermería de pre-grado. La variable principal fue la escucha activa medida por un cuestionario autodirigido. Se emplearon análisis univariado y bivariado mediante las pruebas de $t$ de Student, de ANOVA y de Correlación de Spearman con el programa STATA versión 14. Resultados: el promedio de la escucha activa en los estudiantes fue 50.29. Asimismo, los factores con promedios más altos de escucha activa fueron cursar internado (media $=53.60)$, asistir a prácticas hospitalarias (media $=52.41)$, ser convivientes (media $=51.67)$ o casados (media=51.00), tener un familiar con patología crónica (media=51.46) y recibir capacitación sobre escucha activa o cuidado humanizado (media=51.10). Conclusión: los estudiantes de enfermería tienen un promedio alto de escucha activa. Los factores que se asociaron con la escucha activa fueron ciclo académico, asistencia a prácticas hospitalarias, estado civil, familiar con patología crónica y capacitaciones sobre escucha activa o cuidado humanizado. Los resultados de esta investigación orientan a tomar importancia sobre la escucha activa y los factores que están implicados durante las prácticas clínicas del estudiante. Las casas de estudios deben motivar a sus docentes que fomenten esta habilidad de comunicación en sus estudiantes.
\end{abstract}

Palabras clave: Relaciones Interpersonales; Enfermería Práctica; Estudiantes de Enfermería; Competencia Clínica (Fuente: DeCS).

\begin{abstract}
Objective: to determine what factors are associated with active listening in the clinical practice of nursing students in the Peruvian jungle. Materials and methods: the study was descriptive-correlational cross-sectional design. The population was made up of 85 undergraduate nursing students. The main variable was active listening measured by a self-directed questionnaire. Univariate and bivariate analysis were used using the Student t, ANOVA and Spearman correlation tests with the STATA version 14 program. Results: the average of active listening in students was 50.29 . Likewise, the factors with the highest averages of active listening were attending hospital (mean $=53.60)$, attending hospital practices $($ mean $=$ $52.41)$, living together $($ mean $=51.67)$ or married $($ mean $=51.00)$, having a relative with pathology chronic $($ mean $=$ 51.46) and receive training on active listening or humanized care (mean $=51.10)$. Conclusion: nursing students have a high average of active listening. The factors associated with active listening were academic cycle, attendance at hospital practices, marital status, family with chronic pathology and training on active listening or humanized care. The results of this research aim to take importance on active listening and the factors that are involved during the clinical practices of the student, even the houses of study should motivate their teachers to promote this ability to communicate with their students.
\end{abstract}

Keys words: Interpersonal Relations; Nursing Practical; Students Nursing; Clinical Competence (Source: DeCS).

Para citar:

Ticliahuanca J. Factores asociados a escucha activa durante prácticas clínicas en estudiantes de enfermería de Selva peruana. CASUS. 2019;4(2).62-69.

DOI: $10.35626 /$ casus .2 .2019 .210

\footnotetext{
${ }^{1}$ Hospital Nacional Cayetano Heredia. Bachiller de Enfermería. Correo electrónico: Jticliahuanca1511@gmail.com
}

Fecha de recepción: 11-05-19

Fecha de envío a pares: 15-05-19

Fecha de aprobación por pares : 25-07-19

Fecha de aceptación: 03-08-19 


\section{INTRODUCCIÓN}

La escucha activa es definida según la Clasificación de Intervenciones de Enfermería (CIE), como gran atención y determinación de la importancia de los mensajes verbales y no verbales del paciente (1). Se utiliza una variedad de calificativos en el ámbito clínico respecto a la palabra escucha, que incluyen activa, terapéutica, empática y holística. Cuando se habla de escucha terapéutica se refiere como un proceso interpersonal de confirmación que involucra a todos los sentidos, en el que el terapeuta atiende con empatía todos los mensajes verbales y no verbales del paciente para facilitar la comprensión, la sintesis y la interpretación de la situación del mismo (2).

Asimismo, la escucha impulsa a la independencia y promueve el crecimiento y el desarrollo de los pacientes, de manera que la empatía y la calidez del enfermero resultan necesarios para que se evidencien cambios terapéuticos (3). Sin embargo, existen consecuencias de la mala comunicación enfermero-paciente y estas se inician cuando el enfermero toma el papel del receptor inadecuadamente (4). Hay dos tipos de posibles problemas. El primero está relacionado con la habilidad personal y el segundo con la falta de feedback en la comunicación (4).

Cabe resaltar que la comunicación interpersonal del estudiante de enfermería con el paciente esté posiblemente relacionada con su perfil sociodemográfico o de formación académica. Muchas veces la asistencia a conferencias educativas o a capacitaciones sobre esta temática por parte de los estudiantes de enfermería podría ayudar al desarrollo de sus habilidades comunicacionales con el paciente (5-9). Por otro lado, el manejo de estrategias comunicativas propias del estudiante de enfermería en su desarrollo profesional, mejora cuando reporta mayor experiencia en los establecimientos de salud (10-14). Incluso, la escucha activa suele estar ligada con la práctica continua (15-18). Por otro lado, dentro de la convivencia cercana que se tiene entre amigos, parejas y familiares con alguna enfermedad, surge la comunicación por el interés del bienestar de la persona afectada y por ende se podría adquirir la habilidad de la escucha activa $(16,19-22)$.

En este sentido, se considera que la escucha activa es un componente importante en el cuidado del enfermero, porque es él quien convive mayor tiempo con el paciente hospitalizado y por ende logra su confianza (23). Esto es una realidad que debería tenerse en cuenta para el estudiante desde el inicio de sus prácticas clínicas y posteriormente cuando labore. Lamentablemente, tanto a nivel nacional e internacional y más aún a nivel local esta temática no ha sido muy estudiada y por ello surgió el interés de evaluar a estudiantes de enfermería de una universidad privada de la selva peruana. Cabe resaltar que en comparación con otras regiones del Perú como la Costa, en la Selva no se presentan hospitales con gran demanda de pacientes, lo que conlleva a que los estudiantes presenten ciertas limitaciones en sus prácticas hospitalarias y por ende el contacto con el paciente. Sin embargo, se están aperturando nuevas infraestructuras donde el estudiante podrá tener más contacto con el paciente (24). Por tal motivo, la realización de este estudio permite contribuir científicamente en el campo de enfermería en la región Selva.

Además, es necesario que se conozca cómo se están desarrollando los procesos de escucha activa en los estudiantes, habilidad que les va a permitir generar un clima positivo para la comunicación y las relaciones interpersonales con el paciente en los diferentes servicios de salud (4). Se debe tener en cuenta que existen varios estudios teóricos (25) o cualitativos sobre esta temática, pero muy pocos estudios empíricos, cuantitativos o mixtos. Respecto a lo mencionado anteriormente el objetivo del estudio fue determinar qué factores se asocian a la escucha activa en la práctica clínica de los estudiantes de enfermería de una universidad privada de la selva peruana.

\section{MATERIALES Y MÉTODOS}

El tipo de estudio fue no experimental, con alcance correlacional y de diseño transversal. Se realizó un 
censo de 85 estudiantes de enfermería del cuarto al décimo ciclo de una universidad privada del distrito Nueva Cajamarca de la provincia de Rioja, San Martin en el 2018. Se incluyeron a estudiantes de la carrera de enfermería de ambos sexos del cuarto al décimo ciclo que asistieron a prácticas hospitalarias. Se excluyeron a estudiantes de enfermería que no desearon participar en el estudio y que no completaron los cuestionarios.

Para medir el nivel de escucha activa en los estudiantes de enfermería se utilizó como técnica de recolección el cuestionario autodirigido de "Autoevaluación de la Habilidad de Saber Escuchar". El mismo fue sometido a validez de contenido a través del juicio de 8 expertos, las mismas que fueron sometidas a la prueba Binomial significativa $<0.05$. Asimismo, se realizó una prueba piloto que tuvo como resultado una confiabilidad estadística global con un alfa de Cronbach de 0.68 (26). El cuestionario consta de 20 ítems y cada uno fue valorado a través de la siguiente escala: a menudo ( 3 puntos), a veces (2 puntos), casi nunca (1 punto). Cabe resaltar que 8 items son negativos y el puntaje se invierte. Es una variable cuantitativa y consta de dos dimensiones: física (13 ítems) y psicológica (7 ítems). Además, para medir el nivel de escucha activa del estudiante de enfermería hacia el paciente, se tomó en cuenta el siguiente rango de 0-60. Respecto a la dimensión física el indicador fue de 0 a 39 y la psicológica de 0 a 21 .

Entre los factores sociodemográficos fueron: edad, variable cuantitativa discreta (años); sexo, variable dicotómica (femenino y masculino); ocupación laboral, variable dicotómica (sí y no); estado civil, variable cualitativa politómica (soltero, casado o conviviente); lugar de residencia, variable dicotómica (rural y urbano); religión, variable dicotómica (católico y otra religión); familiar con patología crónica, variable dicotómica (sí y no). Además, se consideraron otras variables de formación tales como estudios técnicos en enfermería, variable dicotómica (sí y no); capacitaciones sobre escucha activa o cuidado humanizado, variable dicotómica (sí y no); ciclo académico, variable dicotómica (primeros ciclos e internado). Cabe resaltar que los primeros ciclos son desde el cuarto al octavo ciclo y el internado corresponde del noveno al décimo ciclo; servicio de rotación, variable cualitativa politómica (emergencia, hospitalización adultos y niños y consultorios externos); asistencia de prácticas hospitalarias, variable dicotómica (asistió a todas y faltó más de una vez).

Para el análisis se empleó el programa STATA versión 14. En el análisis univariado de las variables cualitativas se calcularon frecuencias y porcentajes. Asimismo, para la variable cuantitativas se determinaron la media y la desviación estándar. Por otro lado, en el análisis bivariado entre la escucha activa y sus dimensiones (variables cuantitativas paramétricas) con los factores (variables dicotómicas) se empleó la prueba de t de Student; para analizar los factores de naturaleza politómica se empleó la prueba de ANOVA, por último para la edad (variable cuantitativa no paramétrica) se usó la prueba de Correlación de Spearman. Para todas las pruebas se consideró un nivel de significancia menor o igual a 0.05 .

El estudio fue aprobado por el Comité de Ética de Investigación en Salud de la Universidad Católica Sedes Sapientiae. Asimismo, se realizó la recolección de datos de manera anónima, se explicó a los participantes la finalidad y el uso que se le daría a la información resultante. De igual manera, se garantizó el respeto, la confidencialidad y anonimato de los datos de los encuestados.

\section{RESULTADOS}

En la tabla 1, se muestra que de los 85 estudiantes de enfermería del cuarto al décimo ciclo la mayoría de sexo femenino (75.29\%), el 61.18\% no tenía ocupación laboral, el $88.23 \%$ era soltero, vivía en zona rural $(51.76 \%)$ y reportó la religión católica $(54.12 \%)$. Por otro lado, del total de estudiantes el $81.18 \%$ no tuvo un familiar con patología crónica, el $89.41 \%$ no contó con estudios técnicos en enfermería, el $57.65 \%$ tuvo capacitaciones sobre escucha activa o cuidado humanizado, el $94.12 \%$ cursó los primeros ciclos, el 52.94\% rotó por el servicio de hospitalización 
Tabla 1. Descripción de los estudiantes de enfermería

\begin{tabular}{lcc}
\hline Factores & n & $\%$ \\
\hline $\begin{array}{l}\text { Factores sociodemográficos } \\
\text { Edad a } \\
\text { Sexo }\end{array}$ & $22.72 \pm$ & 4.26 \\
$\quad \begin{array}{l}\text { Masculino } \\
\quad \text { Femenino }\end{array}$ & 21 & 24.71 \\
Ocupación laboral & 64 & 75.29 \\
$\quad$ No & 52 & 61.18 \\
$\quad$ Sí & 33 & 38.82 \\
Estado civil & & \\
$\quad$ Soltero(a) & 75 & 88.23 \\
$\quad$ Casado(a) & 1 & 1.18 \\
$\quad$ Conviviente & 9 & 10.59 \\
Lugar de residencia & & \\
$\quad$ Rural & 44 & 51.76 \\
$\quad$ Urbano & 41 & 48.24 \\
Religión & & \\
$\quad$ Católico(a) & 46 & 54.12 \\
$\quad$ Otra religión & 39 & 45.88 \\
Familiar con patología & & \\
crónica & & \\
$\quad$ No & 69 & 81.18 \\
$\quad$ Sí & 16 & 18.82
\end{tabular}

Factores de formación

académica

Estudios técnicos en

enfermería

$\begin{array}{lcc}\text { No } & 76 & 89.41 \\ \text { Sí } & 9 & 10.59\end{array}$

Capacitaciones sobre escucha

activa o cuidado humanizado

$\begin{array}{lcc}\text { No } & 36 & 42.35 \\ \text { Sí } & 49 & 57.65 \\ \text { o académico } & & \\ \text { Primeros ciclos } & 80 & 94.12 \\ \text { Internado } & 5 & 5.88 \\ \text { vicio de rotación } & & \\ \text { Hospitalización adultos } & 45 & 52.94 \\ \text { Hospitalización niños } & 22 & 25.88 \\ \text { Consultorios externos } & 8 & 9.42 \\ \text { Otros } & 10 & 11.76\end{array}$

Asistencia de prácticas

hospitalarias

\begin{tabular}{lcc} 
Asistió a todas & 79 & 92.94 \\
Faltó más de una vez $^{\text {a }}$ & 6 & 07.06 \\
ucha activa $^{\text {a }}$ & $50.29 \pm 4.00$ \\
mensión física $^{\text {a }}$ & $32.91 \pm 2.78$ \\
mensión psicológica $^{\text {a }}$ & $17.34 \pm 1.85$ \\
\hline
\end{tabular}

adultos y el $92.94 \%$ asistió a todas sus prácticas hospitalarias.

En la tabla 2, se evidencia que los factores estado civil ( $\mathrm{p}=0.011)$, capacitación sobre escucha activa y cuidado humanizado $(\mathrm{p}=0.029)$, ciclo académico $(\mathrm{p}=0.050)$ familiar con patología crónica $(\mathrm{p}=0.040)$
Tabla 2. Análisis bivariado entre los factores y la escucha activa de los estudiantes de enfermería

\begin{tabular}{|c|c|c|c|}
\hline Factores & $\begin{array}{l}\text { Escuc } \\
\text { Media }\end{array}$ & $\begin{array}{c}\text { activa } \\
\text { DE }\end{array}$ & p-valor \\
\hline \multicolumn{4}{|l|}{$\begin{array}{l}\text { Factores } \\
\text { sociodemográficos }\end{array}$} \\
\hline Edad* & \multirow{2}{*}{\multicolumn{2}{|c|}{0.02}} & 0.85 \\
\hline Sexo & & & 0.46 \\
\hline Masculino & 50.85 & 3.38 & \\
\hline Femenino & 50.11 & 4.20 & \\
\hline Ocupación laboral & & & 0.72 \\
\hline No & 50.17 & 4.23 & \\
\hline Sí & 50.48 & 3.68 & \\
\hline Estado civil & & & 0.01 \\
\hline Soltero (a) & 50.17 & 3.64 & \\
\hline Casado (a) & 51.00 & 0.00 & \\
\hline Conviviente & 51.67 & 6.52 & \\
\hline Lugar de residencia & & & 0.87 \\
\hline Rural & 50.23 & 3.98 & \\
\hline Urbano & 50.37 & 4.08 & \\
\hline Religión & & & 0.89 \\
\hline Católico(a) & 50.24 & 4.32 & \\
\hline Otra religión & 50.36 & 3.65 & \\
\hline $\begin{array}{l}\text { Familiar con patología } \\
\text { crónica }\end{array}$ & & & 0.04 \\
\hline No & 48.56 & 4.17 & \\
\hline Sí & 51.46 & 3.97 & \\
\hline $\begin{array}{l}\text { Factores de formación } \\
\text { académica }\end{array}$ & & & \\
\hline $\begin{array}{l}\text { Estudios técnicos en } \\
\text { enfermería }\end{array}$ & & & 0.46 \\
\hline No & 50.18 & 3.69 & \\
\hline Sí & 51.22 & 6.26 & \\
\hline Recibió capacitación & & & 0.02 \\
\hline No & 49.19 & 3.90 & \\
\hline Sí & 51.10 & 3.92 & \\
\hline Ciclo académico & & & 0.05 \\
\hline Primeros ciclos & 50.09 & 3.99 & \\
\hline Internado & 53.60 & 2.70 & \\
\hline Servicio de rotación & & & 0.36 \\
\hline $\begin{array}{l}\text { Hospitalización } \\
\text { adultos } \\
\text { Hospitalización }\end{array}$ & 50.60 & 4.19 & \\
\hline niños & 49.77 & 4.12 & \\
\hline $\begin{array}{l}\text { Consultorios } \\
\text { externos }\end{array}$ & 50.5 & 2.33 & \\
\hline Otros & 49.9 & 4.33 & \\
\hline $\begin{array}{l}\text { Asistencia de prácticas } \\
\text { hospitalarias }\end{array}$ & & & 0.05 \\
\hline Asistió a todas & 52.41 & 3.81 & \\
\hline $\begin{array}{l}\text { Faltó más de una } \\
\text { vez }\end{array}$ & 48.66 & 6.25 & \\
\hline
\end{tabular}

y asistencia a prácticas hospitalarias $(\mathrm{p}=0.050)$ se relacionaron significativamente con la escucha activa. El promedio más alto de escucha activa correspondió al estado civil conviviente (media= 
51.67) y casados (media $=51.00$ ), estudiantes que recibieron capacitación sobre escucha activa $($ media $=51.10)$, cursar internado $($ media $=53.60)$, tener un familiar con patología crónica (media=51.46) y asistir a prácticas hospitalarias (media $=52.41)$.

Por otro lado, en la tabla 3, se observó que los factores que se relacionaron significativamente con la dimensión física de la escucha activa fueron el estado civil ( $\mathrm{p}=0.017)$, la capacitación en escucha activa o cuidado humanizado $(\mathrm{p}=0.048)$ y el ciclo académico $(\mathrm{p}=0.050)$, en donde resultó que el promedio más alto se observó en el estado civil conviviente $\quad($ media $=33.89)$ y casados (media $=33.00$ ), en recibir capacitación en escucha activa y cuidado humanizado (media $=33.43$ ) y en el ciclo académico internado (media $=35.2)$. El resto de asociaciones no fue significativo.

\section{DISCUSIÓN}

Los factores asociados a la escucha activa en la práctica clínica de los estudiantes dentro de los sociodemográficos fueron el estado civil y tener un familiar con patología crónica, y respecto a los de formación académica fueron el recibir capacitación en escucha activa o cuidado humanizado, ciclo académico y asistencia a prácticas hospitalarias.

Según el resultado, los estudiantes que convivieron o estuvieron casados tuvieron promedios más altos de la escucha activa. La literatura no reporta semejanza con este hallazgo, no obstante un estudio realizado en parejas colombianas mostró que lograron consolidar un modo de relación comunicativa basado en la escucha activa, la crítica correctiva balanceada con la estima y el respeto (20). Otro estudio realizado en hombres y mujeres mostró que uno de los estilos de comunicación más importantes tanto en la persona misma como en la pareja es el social afiliativo, es decir, hacer uso de expresiones de cariño y afecto como formas de complacer y ser sociable con la pareja facilitan la mutua comprensión, el respeto y la toma responsable de decisiones en la pareja (21). Asimismo, la escucha activa tiene el poder transformador tanto para el emisor como para el receptor (22). Puesto que ayuda a que el emisor tenga la capacidad de poder desarrollar la escucha
Tabla 3. Análisis bivariado entre los factores y las dimensiones de la escucha activa de los estudiantes de enfermería

\begin{tabular}{|c|c|c|c|c|c|c|}
\hline \multirow[t]{2}{*}{ Factores } & \multicolumn{3}{|c|}{ Dimensión física } & \multicolumn{2}{|c|}{$\begin{array}{l}\text { Dimensión } \\
\text { psicológica }\end{array}$} & \multirow[b]{2}{*}{$\mathbf{p}$} \\
\hline & $\mathbf{m}$ & DE & $\mathbf{p}$ & $\mathbf{m}$ & DE & \\
\hline \multicolumn{7}{|l|}{ Factores } \\
\hline sociodemográficos & & & & & & \\
\hline Edad* & \multicolumn{2}{|c|}{0.01} & 0.90 & \multicolumn{2}{|c|}{0.03} & 0.73 \\
\hline Sexo & & & 0.48 & & & 0.70 \\
\hline Masculino & 33.29 & 2.57 & & 17.48 & 1.75 & \\
\hline Femenino & 32.79 & 2.86 & & 17.30 & 1.87 & \\
\hline Ocupación laboral & & & 0.89 & & & 0.48 \\
\hline No & 32.88 & 2.92 & & 17.23 & 1.95 & \\
\hline Sí & 32.97 & 2.60 & & 17.52 & 1.66 & \\
\hline Estado civil & & & 0.01 & & & 0.29 \\
\hline Soltero (a) & 32.84 & 2.54 & & 17.29 & 1.79 & \\
\hline Casado (a) & 33.00 & 0.00 & & 17.00 & 0.00 & \\
\hline Conviviente & 33.89 & 4.43 & & 18.78 & 2.33 & \\
\hline Lugar de residencia & & & 0.79 & & & 0.99 \\
\hline Rural & 32.84 & 2.93 & & 17.34 & 1.72 & \\
\hline Urbano & 33.00 & 2.66 & & 17.34 & 1.97 & \\
\hline Religión & & & 0.98 & & & 0.84 \\
\hline Católico(a) & 32.91 & 2.96 & & 17.30 & 2,01 & \\
\hline Otra religión & 32.92 & 2.61 & & 17.38 & 1.63 & \\
\hline $\begin{array}{l}\text { Familiar con } \\
\text { patología crónica }\end{array}$ & & & 0,44 & & & 0,60 \\
\hline No & 33.03 & 2.92 & & 17.39 & 1.67 & \\
\hline Sí & 32.44 & 2.16 & & 17.13 & 2.47 & \\
\hline $\begin{array}{l}\text { Factores de formación } \\
\text { académica }\end{array}$ & & & & & & \\
\hline $\begin{array}{l}\text { Estudios técnicos en } \\
\text { enfermería }\end{array}$ & & & 0.47 & & & 0.57 \\
\hline No & 32.84 & 2.60 & & 17.30 & 1.74 & \\
\hline Sí & 33.56 & 4.19 & & 17.67 & 2.65 & \\
\hline Recibió capacitación & & & 0.04 & & & 0.06 \\
\hline No & 32.22 & 2.64 & & 16.92 & 1.93 & \\
\hline Sí & 33.43 & 2.81 & & 17.65 & 1.71 & \\
\hline Ciclo académico & & & 0.05 & & & 0.18 \\
\hline Primeros ciclos & 32.78 & 2.76 & & 17.28 & 1.86 & \\
\hline Internado & 35.2 & 2.39 & & 18.4 & 1.14 & \\
\hline Servicio de rotación & & & 0.60 & & & 0.12 \\
\hline $\begin{array}{l}\text { Hospitalización } \\
\text { adultos }\end{array}$ & 32.96 & 2.92 & & 17.58 & 1.76 & \\
\hline Hospitalización & & & & & & \\
\hline niños & 32.73 & 2.43 & & 17.05 & 2.32 & \\
\hline $\begin{array}{l}\text { Consultorios } \\
\text { externos }\end{array}$ & 33.5 & 2.51 & & 17.00 & 1.19 & \\
\hline Otros & 32.7 & 3.43 & & 17.2 & 1.39 & \\
\hline Asistencia de prácticas & & & & & & \\
\hline hospitalarias & & & 0.25 & & & 0.63 \\
\hline $\begin{array}{l}\text { Asistió a todas } \\
\text { Faltó más de una }\end{array}$ & 33.01 & 2.68 & & 17.36 & 1.80 & \\
\hline vez & 31.67 & 4.03 & & 17 & 2.36 & \\
\hline
\end{tabular}

activa fuera de su relación haciéndola presente dentro de sus competencias laborales y mejorando 
la interacción que se tenga con otras personas tanto a nivel profesional como laboral (22). En este sentido esta experiencia comunicativa de los estudiantes que tienen una pareja repercute en sus prácticas clínicas con los pacientes.

Otro hallazgo fue que los estudiantes de enfermería que tuvieron un familiar con alguna patología presentaron una mayor escucha activa en comparación a los que no. La literatura no reporta semejanza con este hallazgo; sin embargo un estudio muestra que vivir o cuidar a un familiar enfermo repercute negativamente en el cuidador o la persona más cercana, donde él percibe la enfermedad como adquisición de una serie de sentimientos como soledad, tristeza, culpa $\mathrm{y}$ lástima e incluso es propenso a sufrir depresión. Pero admiten que en la vivencia con el familiar enfermo conocen el inicio de la enfermedad, sus atributos, las formas de tratamiento, entre otros (19). Es por ello que el estudiante de enfermería teniendo la experiencia de vivir con un familiar enfermo evidencia seguridad al comunicarse con un paciente por el conocimiento y la sensibilidad que adquirió a través de esta experiencia familiar (16).

Los estudiantes que asistieron a una capacitación sobre escucha activa o cuidado humanizado tuvieron una mayor escucha activa hacia al paciente. Un estudio realizado en estudiantes de enfermería de China mostró que se asoció significativamente la experiencia de capacitación clínica y las habilidades de comunicación (5). Asimismo, otro estudio realizado en ese mismo país evidenció que los estudiantes que asistieron a un curso de capacitación fue efectivo para mejorar las habilidades de comunicación, especialmente en términos del contenido y el manejo de las barreras de comunicación (6). Otro estudio mostró que los estudiantes estuvieron satisfechos con las actividades de evaluación por pares (mediante videos) y apreciaban la contribución a sus habilidades de comunicación. Además, en el análisis de la retroalimentación de los pares se reveló que la comunicación de los estudiantes se volvió gradualmente más centrada hacia el paciente (7). Una explicación de este hallazgo es que en el campo clínico se necesita de un personal de enfermería cada vez más capacitado tanto en el conocimiento (en conceptos y reflexiones científicas que instiguen el progreso teórico) como de las habilidades (práctica) de forma que "el saber lleve a eficiencia en el hacer" $(8,9)$. Es importante resaltar que el desarrollo de los programas de capacitaciones es una opción de gran valor que debe ser tomada por los enfermeros y estudiantes de enfermería para estimular la razón crítica que conlleve al desarrollo de construcción y reconstrucción del conocimiento en su práctica profesional (9).

Los resultados también fueron satisfactorios respecto a la escucha activa y la dimensión física para el grupo de estudiantes que se encontraban en el nivel de internado que corresponde al noveno y décimo ciclo de la carrera de enfermería en comparación con ciclos inferiores. Al respecto, un estudio realizado en estudiantes de enfermería mostró una correlación significativa entre el ciclo educativo de los estudiantes y el comportamiento de comunicación clínica y de habilidad de comunicación de tratamiento. Es decir, los estudiantes de ciclos superiores tenían mejores habilidades de comunicación en comparación con ciclos inferiores (10). Asimismo, un estudio realizado en Lima en internos de enfermería reportó una evolución favorable en el desarrollo de sus habilidades de comunicación comparando el inicio $(0 \%)$ y el fin de internado (8.1\%) (11). Esto sería debido a que los estudiantes de ciclos inferiores tienden a experimentar situaciones de estrés por la nueva experiencia en un campo desconocido. De manera que,su atención exige un esfuerzo físico que les causa dificultad en la comunicación, sentimientos de ansiedad e inseguridad (12). Además, la transición del enfermero novato a experto requiere de la experiencia y en forma relevante involucrarse emocionalmente con el paciente y su familia; siendo este aspecto el que le permite encajar los elementos de la situación y ser capaz de identificar lo que sucede sin perder tiempo en soluciones y diagnósticos alternativos (13). Por ende, la comunicación terapéutica entre estudiantes y 
pacientes de enfermería requieren de educación y práctica clínica (14).

Dentro de los hallazgos los estudiantes que asistieron a todas sus prácticas hospitalarias tuvieron promedios mayores de escucha activa en comparación con los estudiantes que faltaron más de una vez. Este resultado coincide con lo expuesto por un estudio cualitativo realizado en estudiantes de enfermería que asistieron a todas sus prácticas clínicas donde los estudiantes consideraron que aprender a escuchar es un acto de cuidado (16). Otro estudio realizado en estudiantes reportó que la percepción del dolor del paciente en las prácticas clínicas hace consciente su falta de "experiencia", y reconocen que con el tiempo y la práctica lograrán adquirir las destrezas que les permitan demostrar eficacia en el cuidado del paciente (17). Otra explicación del hallazgo es que los estudiantes de enfermería que asisten a las prácticas clínicas lo hacen para desarrollar una serie de habilidades de comunicación (16). Es decir, se conectan con los pacientes y hacen una relación interpersonal cara a cara a través de la empatía y las habilidades de escucha, la cual, es adquirida en el transcurso de la práctica que el estudiante de enfermería realiza en cada oportunidad de interacción diaria con el paciente (17). Cabe resaltar que estas habilidades se adquieren sólo con las prácticas hospitalarias y las enseñanzas del docente de práctica (17), pero lamentablemente hay muchos estudiantes que desertan a sus prácticas y les dificulta desarrollar estas habilidades. Uno de los motivos es por el temor de experimentar ansiedad y otras cargas emocionales (18).
Entre las limitaciones se encontraron la poca población y la inclusión de ciertos factores como tipo de universidad, percepciones del estudiante frente a la labor docente, y características clínicas del paciente, entre otros que deberían tomarse en cuenta para futuros estudios cuantitativos y mixtos. Cabe destacar que la novedad de este estudio fue considerar a los estudiantes de enfermería de la selva peruana, lugar en el que no se encontró evidencia científica sobre la temática propuesta.

\section{CONCLUSIONES}

El estudio concluye que los estudiantes de enfermería estudiados tienen un alto promedio de escucha activa. Además, dentro de los factores de formación con promedios altos de escucha activa en la práctica clínica se destacan: la asistencia a todas sus prácticas hospitalarias, recibir capacitaciones de escucha activa y/o cuidado humanizado y cursar el ciclo académico internado. Mientras que los factores sociodemográficos con promedios más altos de escucha activa son convivir o estar casados y tener un familiar con una patología crónica.

A partir de estos hallazgos se deben fomentar las capacitaciones sobre escucha activa, implementarlas o dar mayor importancia en la malla curricular de la carrera de enfermería, porque los aprendices de enfermería tienen que adquirir diversas competencias clínicas, una de las más importantes es la competencia de comunicación terapéutica con el paciente.

\section{REFERENCIAS BIBLIOGRÁFICAS}

1. MccloskeY J, Bulecheck G. Clasificación de Intervenciones de 3. Rogers CR: The necessary and Enfermería (CIE). 3aed. Madrid: Harcourt; 2001.

2. Kemper BJ: Therapeutic listening: developing the concept. Journal of Psychosocial Nursing and Mental 4 Health Services. 1992;30(7):2123. sufficient conditions of therapeutic personality change. Journal of Consulting Psychology. 5 . 1957;21:95-103.

Van-der Hofstadt Román CJ, Quiles Marcos Y, José QSM. Técnicas de comunicación para profesionales de enfermería,
Conselleria de Sanitat. Primera ed. Valencia: Generalitat Valenciana; 2006.

Jianfei X, Siqing D, Chunmei W,
Aizhong L. Articule: An
evaluation of the communication
skills of nursing students during
practical clinical training. Nurse
Education Today. 2013;33:823-
827.


6. $\quad$ Ying L, Wenru W. Articule: Development and evaluation of a learner-centered training course on communication skills for baccalaureate nursing students. Nurse Education Today. 2013;33:1617-1623.

7. Chin Y. Articule: Training nursing students' communication skills with online video peer assessment. Computers \& Education. 2016;97:21-30.

8. MsC. Barroso Z; Colomer E. Las Competencias: su importancia para la planificación de los programas de capacitación de Enfermería. Revista Cubana de Enfermería. 2007.

9. Mador DD, Gomes IP, Coutinho SED, Costa TNA, Collet N. A vivência do cuidado em oncologia pediátrica e a busca pela produção do conhecimento. Rev enferm UFPE on line 2010; 4(2): 666-72. Disponible en: http://www.revista.ufpe.br/revistae nfermagem/index.php/revista/artic le/view/851.

10. Shafakhah M, Zarshenas L, Sharif $\underline{F}$, Sarvestani RS. Evaluation of Nursing Students' Communication Abilities in Clinical Courses in Hospitals. Global Journal of Health Science. 2015;7(4): 323328.

11. Arévalo JM. Evolución de Habilidades Sociales en Internos de Enfermería de una Universidad Privada de Lima. Ciencia y arte de Enfermería. 2017; 2(1).

12. Silva L; Mara E. Reações comunicativas dos alunos de enfermagem frente ao primeiro 19. Muñoz LA, Price YM, Reyes M, estágio curricular. Revista da Escola de Enfermagem da USP. 2008; 42(4).

13. Benner P. Práctica progresiva en enfermería. Manual de comportamiento profesional. Barcelona: Grijalbo; 1987:58-78.

14. Weissman GV. Evaluar la autoeficacia de los estudiantes de enfermería de grado asociado en habilidades de comunicación y actitudes en el cuidado del paciente moribundo. Enseñanza y aprendizaje en enfermería. 2011;6(2):64-72. doi: 10.1016 / j.teln.2010.10.004.

15. Valderrama ML, Peña AP, Clavijo LM. Narrativa: el estudiante de enfermería aprendiendo el arte de cuidar. Rev Cuidarte. 2017;8(1):1488-98.

16. Henao M, Fajardo E, Núñez ML. La experiencia de estudiantes de enfermería ante el dolor infligido en la práctica clínica. Salud Uninorte [Internet]. 2014;30(3):431-441. Disponible en:

http://www.redalyc.org/articulo.oa ?id=81737153005.

17. Rosenberg S, Gallo-Silver L. Habilidades de comunicación terapéutica y estudiantes de enfermería en el entorno clínico. Enseñanza y aprendizaje en enfermería. 2011; 6 (1): 2-8. doi: 10.1016 / j.teln.2010.05.003.

18. Melincavage S. Student nurses' experiences of anxiety in the 26. Suarez C.A. Nivel de aplicación clinical setting. Nurse Education Today 2011;31(8):785-789.
Ramírez M, Costa M. The experience of family caregivers of older people with depression. Revista da escola de enfermagem da usp. 2010; 44(1).

20. Cuervo JJ, Izquierdo C, Anguera MT, Linares JL. Comunicación y perdurabilidad en parejas viables. Estudio observacional de caso múltiple [Tesis doctoral]. Barcelona: Universidad Autónoma de Barcelona;2014.

21. Flores MM. Comunicación conflicto: ¿Qué tanto impactan en la satisfacción marital?. Acta de Investigación Psicológica. 2011;1(2).

22. Naranjo MG. Escucha activa y empática. Editorial Elearning, SL;2018.9.

23. Mirón R. Comunicación de malas noticias: perspectiva enfermera. Rev Esp Com Sal. 2010;1(1): 3949.

24. Gobierno Regional de San Martín. Ratifican apertura del hospital de Moyobamba para el 20 de julio 2019 [internet]. San Martín.Perú; 2019. Disponible en https://web.regions anmartin.gob.p e/WebApp/Noticias?url=noticia\&i $\underline{\mathrm{d}=5688}$.

25. Snyder M, Lindquist R. Terapias complementarias y alternativas en enfermería. 1a ed en español, 6ta ed en inglés. México: Editorial El Manual Moderno; 2010. de la escucha activa en la práctica clínica por los estudiantes de enfermería de la UNMSM, 2008. 